\title{
FRACTURAS DE ESPINAS TIBIALES EN LA EDAD PEDIÁTRICA: REVISIÓN
}

\author{
Darás Ballester, Álvaro; SalomTaverner, Marta
}

Objetivo de la revisión: Pese a que las fracturas de espinas tibiales son poco frecuentes, su importancia debe ser tenida en cuenta debido a su proximidad a la fisis proximal de tibia, así como su relación con la inserción de ligamento cruzado anterior, uno de los más importantes estabilizadores de la rodilla. Esta revisión tiene como objetivo sintetizar los conceptos acerca del diagnóstico, manejo y pronóstico de dichas lesiones descritos por la literatura más actualizada.

Conceptos novedosos: la literatura actual describe la existencia de diferentes modalidades de tratamiento tanto conservador como quirúrgico, sin determinar claras diferencias entre ellas a largo plazo. No obstante, se establecen criterios para orientar el tratamiento de las mismas en función de las características del paciente y de la fractura.

Resumen: las fracturas de espinas tibiales en la edad pediátrica son lesiones poco frecuentes. Generalmente es la clínica y el mecanismo lesional los que hacen sospechar estas fracturas, y el diagnóstico definitivo se consigue con pruebas de imagen, siendo necesaria, en ocasiones, la realización de TC O RM. En cuanto al tratamiento de estas fracturas, no existe un consenso claro, debido a la gran variedad de opciones terapéuticas, pero sí puede afirmarse que el tratamiento de elección para las fracturas desplazadas es la reducción y fijación de forma quirúrgica. 


\section{INTRODUCCIÓN}

La fractura-avulsión de espina tibial es una lesión poco frecuente en niños (aproximadamente se da en 3 de cada 100.000 niños al año), supone alrededor del $2 \%$ del total de lesiones de rodilla en la infancia, y tiene un pico de incidencia entre los 8 y los 14 años ${ }^{(1-8)}$.

Entre las principales causas de estas lesiones encontramos los accidentes deportivos, generalmente caídas de bicicleta. Habitualmente, el mecanismo lesional es el mismo que produce una lesión del Ligamento Cruzado Anterior (LCA) en un paciente adulto: una rotación de la tibia respecto al fémur y un hiperextensión forzada de la articulación $(1,2)$.

La mayor parte de las veces la lesión se produce en la zona de la eminencia intercondílea anterior (con una frecuencia de $10: 1$ respecto a la posterior) ${ }^{(1-7)}$.

Estas fracturas pueden asociar lesiones ligamentosas (Ligamento Cruzado Anterior - Ligamento Colateral Medial preferentemente) y meniscales, por lo que en la evaluación inicial deben ser valoradas y descartadas, ya que si pasan desapercibidas pueden producir laxitud o inestabilidad residual a largo plazo ${ }^{(1-7)}$.

\section{ANATOMÍA QUIRÚRICA}

La eminencia intercondílea es la región de la superficie proximal de la tibia que se encuentra situada entre los platillos tibiales. Dentro de la misma, encontramos una eminencia anterior (entre los polos anteriores de ambos meniscos) y una posterior ${ }^{(1)}$.
Además, dentro de la eminencia intercondílea encontramos dos tuberosidades o espinas (medial y lateral).

En su inserción distal, el LCA presenta dos fascículos (anterior y lateral), que se insertan respectivamente en la zona anterior de la espina tibial medial y en el cuerno anterior del menisco medial. Por su parte, el LCP no se inserta en las espinas tibiales, sino justo en la zona posterior de la eminencia tibial $^{(1)}$ (Figura 1).

En el esqueleto inmaduro, la superficie de la eminencia intercondílea se encuentra cubierta íntegramente por cartílago ${ }^{(1)}$.
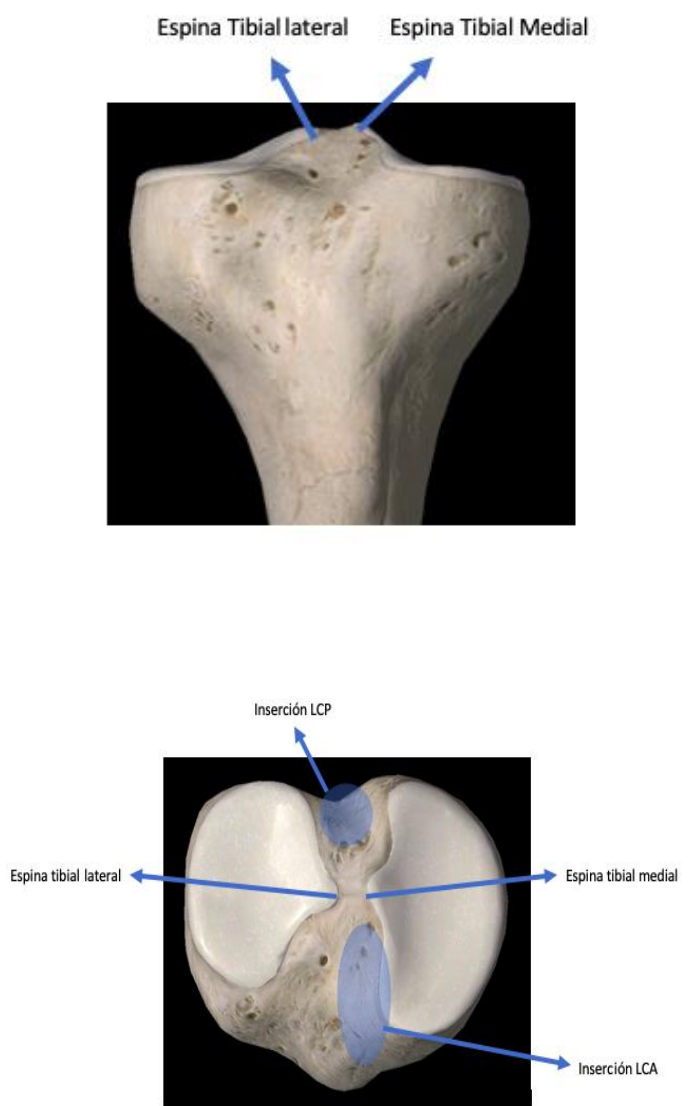

Figura 1: anatomía de la eminencia intercondílea, donde podemos ver referenciadas las inserciones de LCA y LCP. 
En el esqueleto inmaduro, la espina tibial presenta una mayor debilidad que el LCA, ya que no se encuentra completamente osificada, por lo que la misma sobrecarga que puede producir una lesión aislada de LCA en un adulto, en un niño puede producir un fracaso del hueso esponjoso subcondral, produciendo la avulsión de la espina tibial ${ }^{(1-5)}$ (Figura 2).

Debido a este mismo mecanismo de acción, se ha descrito un porcentaje del 29 al 35\% de atrapamientos del menisco medial bajo el fragmento avulsionado, lo cual puede dificultar la reducción del mismo ${ }^{(1,2)}$.

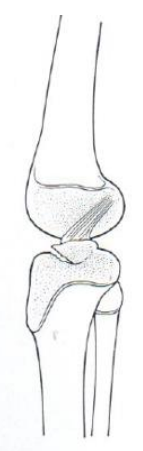

Figura 2: al presentar la espina tibial una mayor debilidad que el LCA, el mecanismo de lesión que produciría una lesión del LCA en el adulto puede provocar avulsión de la espina tibial.

\section{CLASIFICACIÓN}

La clasificación más empleada es la que propusieron Meyers y McKeever en 1959, basada en el grado de desplazamiento, y posteriormente modificada por Zaricznyj ${ }^{(1-}$ 3,8,9) (Figura 3):

TIPO I: fractura mínimamente desplazada.

TIPO II: se observa desplazamiento de tercio anterior, produciendo un levantamiento tipo "bisagra" del fragmento.
TIPO III: se observa una separación completa del fragmento.

TIPO IV (añadida posteriormente por Zaricznyj): como la tipo III pero con rotación del fragmento o importante conminución.

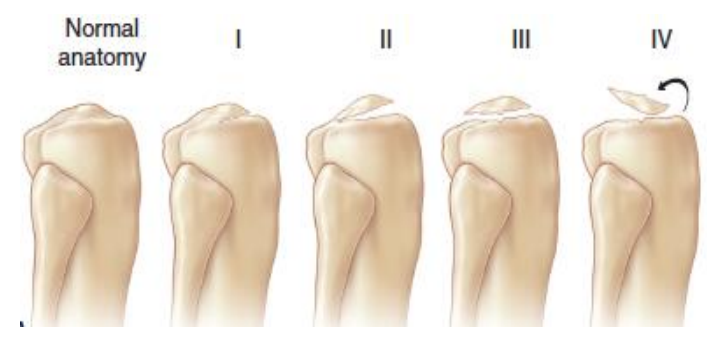

Figura 3: Clasificación de Meyers y

Mckeever, modificada por Zariczny ${ }^{(1)}$

\section{EVALUACIÓN CLÍNICA}

Por lo general, encontraremos un paciente que presenta, tras un mecanismo de torsión de la tibia sobre el fémur con extensión de rodilla asociada, importante dolor a nivel de rodilla, que limita el rango de flexoextensión activa y pasiva e imposibilita la carga sobre el miembro afecto. Por tanto, el paciente rechazará la marcha. Es muy típico encontrar una actitud de semiflexión de rodilla, y los intentos de extensión pasiva producirán intenso dolor.

También es frecuente encontrar derrame articular, que, en caso de artrocentesis evacuadora, consistirá fundamentalmente en hemartros ${ }^{(1,2,8)}$.

En cuanto a las maniobras para valorar la estabilidad articular, serán difíciles de realizar en el momento agudo, pero puede ser útil, en una primera exploración, descartar las lesiones que se asocian con más frecuencia a este tipo de fracturas, que son la lesión a nivel de LCM y LCA ${ }^{(1-8)}$. 


\section{EXPLORACIONES}

\section{COMPLEMENTARIAS}

La primera prueba de imagen que ofrecerá información será la radiografía simple anteroposterior y lateral de rodilla, siendo más fácil observar este tipo de lesiones en la proyección lateral (Figura 4). En ocasiones, el fragmento avulsionado puede estar compuesto fundamentalmente por cartílago no osificado y una delgada fracción osificada, por lo que puede la lesión puede ser infraestimada, o incluso pasar desapercibida. Otra proyección radiológica de utilidad es la proyección anteroposterior con inclinación paralela a superficie articular tibial ${ }^{(1-4,8,9)}$.

Es importante destacar que si la exploración física sugiere una lesión de ligamentos colaterales o una fractura fisaria, pueden ser útiles las radiografías en estrés o sobrecarga bajo sedación, aunque este punto se encuentra actualmente en controversia, de hecho, en nuestro centro no se realizan este tipo de pruebas de imagen, considerando más útil y menos agresiva la realización de RM.

La realización de Tomografía Computarizada (TC) puede ser útil para ampliar el estudio diagnóstico y tipificar la fractura, sobre todo si el grado de desplazamiento no puede ser valorado con claridad en la radiografía lateral, para realizar una adecuada planificación quirúrgica en los casos de fracturas desplazadas, así como para valorar la congruencia articular tras una reducción cerrada en caso de fracturas tipo II $(1,2,6,8)$.

Como ya se ha dicho, otra prueba diagnóstica de utilidad es la Resonancia Magnética (RM), que puede ser de gran valor para identificar posibles lesiones de partes blandas asociadas, preferentemente en pacientes con fracturas no desplazadas que no van a someterse a intervención quirúrgica, así como para identificar fragmentos puramente cartilaginosos que puedan pasar desapercibidos en las radiografías o TC $(1,2,6,8)$.

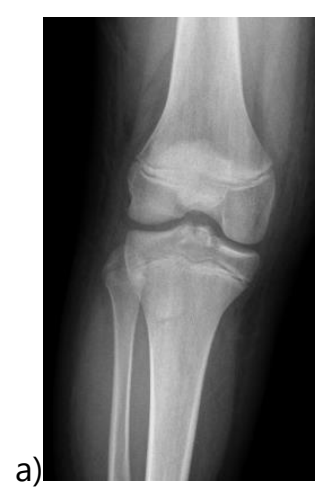

b)

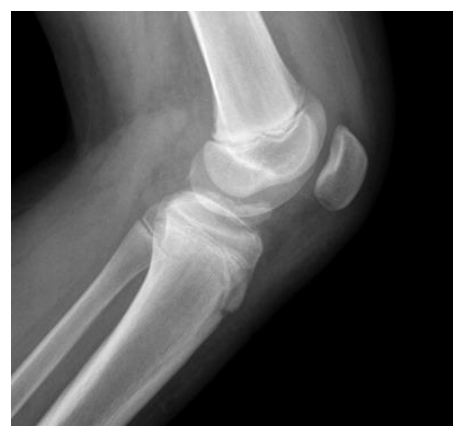

Figura 4: Radiografía anteroposterior (a) y lateral (b) en paciente con fractura de espinas tibiales. En la proyección lateral se aprecia con mayor claridad la fractura.

\section{TRATAMIENTO}

La decisión terapéutica se basa en el tipo de fractura según la clasificación de Meyers y McKeever ${ }^{(1-12)}$.

Tipo I: en este tipo de fracturas, en las que el desplazamiento del fragmento es mínimo, el tratamiento de elección es ortopédico, mediante la colocación de un yeso cerrado isquiopédico en extensión de rodilla. Previamente a la inmovilización, es importante el drenaje del hemartros, si este 
existe, para reducir el dolor y favorecer la posición en extensión de rodilla. Respecto a la extensión completa o no de la rodilla, existe controversia en la literatura, ya que existen autores que defienden la inmovilización en completa extensión ${ }^{(1,6,8)}$, ya que esta posición permite un contacto entre los cóndilos femorales y el fragmento avulsionado, que favorecerá que se mantenga la reducción de la fractura, y otros defienden la inmovilización a 10-20 de flexión de rodilla para favorecer una correcta tensión del LCA y evitar laxitud posterior ${ }^{(2,8)}$.

Tras la inmovilización, el seguimiento suele realizarse cada 2 semanas hasta la retirada del yeso a las 6 semanas.

Tipo II: son aquellas fracturas en las que encontramos un desplazamiento anterior de la fractura. Ante estas fracturas, el tratamiento inicial, descrito en su momento por Meyers y Mckeever, es ortopédico, mediante reducción cerrada mediante una maniobra de hiperextensión de rodilla, habiendo realizando previamente una artrocentesis evacuadora, e inmovilización mediante yeso isquiopédico en extensión o ligera flexión de rodilla (siguiendo los principios de las fracturas tipo I).

No obstante, cabe la posibilidad de no obtener una correcta reducción mediante maniobras cerradas o de que exista un posterior desplazamiento de la fractura, debido generalmente a interposición del ligamento intermeniscal $o$ del cuerno anterior del menisco medial, por lo que en estos casos estaría indicada la reducción abierta o artroscópica de la fractura y posterior inmovilización mediante yeso cerrado en extensión de rodilla o ligera flexión. Los resultados descritos son similares en cuanto a los dos tipos de tratamiento.

El seguimiento de este tipo de fracturas es muy similar a las Tipo I, y es reseñable que, en ocasiones, puede ser conveniente la realización de un TC de control para valorar la correcta reducción, e incluso se puede plantear la realización RM para descartar lesiones meniscales $O$ ligamentosas asociadas.

Tipo III y IV: se trata de fracturas en las que existe un desplazamiento significativo del fragmento. La reducción cerrada no suele ser eficaz en estos casos, por lo que se opta por una reducción abierta de la fractura, que permite la limpieza del hematoma y posibles restos condrales, así como la inspección de los cuernos anteriores de los meniscos y la extracción del menisco si se encuentra interpuesto e impidiendo la reducción del fragmento.

La principal controversia en cuanto al tratamiento quirúrgico de estas fracturas se encuentra en si es más conveniente la realización de una cirugía abierta (artrotomía) o de una cirugía artroscópica. En la literatura se han descrito resultados excelentes para ambos procedimientos, siendo los resultados equivalentes entre ellos.

No obstante, existen ventajas e inconvenientes descritos para cada unos de los procedimientos, habiéndose descrito, por ejemplo, una menor tasa de complicaciones quirúrgicas, tiempo de hospitalización y dolor postoperatorio en los pacientes sometidos a artroscopia $(1,2,7,8,10-12)$, así como unas menores tasas de artrofibrosis en los pacientes sometidos a artrotomía, ya que se ha visto que existe un riesgo mayor de artrofibrosis según el 
tiempo que pasa desde la lesión hasta la intervención, y normalmente, los pacientes esperan menor tiempo si se intervienen mediante artrotomía $a^{(2,4,7,8,9,12) \text {. }}$

Además, dentro del tratamiento quirúrgico, existen también dos procedimientos descritos para la osteosíntesis del fragmento: tornillos canulados o suturas transóseas; habiéndose descrito resultados satisfactorios para ambas opciones.

Posteriomente a la osteosíntesis, se realiza una inmovilización durante 4 semanas. Diversos autores defienden la movilización precoz a partir de las 4 semanas para evitar la artrofibrosis ${ }^{(4,5,6,7,7,8,12)}$.

A continuación, procedemos a describir ambas técnicas quirúrgicas descritas:

Artrotomía: generalmente se realiza un pequeño abordaje anteromedialpararrotuliano. Se realiza una inspección de la articulación, que permite evacuar el hematoma formado y los restos condrales, así como la reducción del fragmento. Posteriomente, se realiza la técnica de osteosíntesis, que puede variar en función de las características del paciente $o$ del fragmento avulsionado:

En el niño pequeño, es frecuente encontrar un fragmento voluminoso compuesto por cartílago fundamentalmente, por lo que tras la reducción se puede optar por sintetizar el framento mediante una sutura periférica absorbible directamente sobre el lecho fracturario.

En el adolescente o si encontramos un fragmento más pequeño y de mayor consistencia ósea, la opción de sutura atroscópica es muy atractiva: consiste en entrelazar una sutura no reabsorbible a través de la porción distal de LCA, realizando una sutura tipo Krakow, y extrayéndola por el propio fragmento avulsionado de espina tibial. Acto seguido, a partir de un punto de entrada anteromedial sobre la metáfisis tibial, se perforan dos orificios de manera oblicua dirigidos hacia el lecho medial de la epífisis tibial (en sentido distal-proximal), evitando cuidadosamente la fisis. A continuación, se reubica el fragmento en su lecho, extrayendo las suturas a través de los túneles óseos realizados y anudando en la cara anteromedial de metáfisis tibial (Figura 5).

En pacientes esqueléticamente más maduros, en los que la integridad de la fisis no preocupa tanto, y en los que el fragmento osteocondral tiene un tamaño suficiente $(>1 \mathrm{~cm})$, se puede fijar la espina tibial con un tornillo de minifragmentos dirigido hacia la metáfisis. Esta técnica requiere un adecuado fragmento osteocondral y una suficiente amplitud de la epífisis para no dañar la fisis (Figura 6).

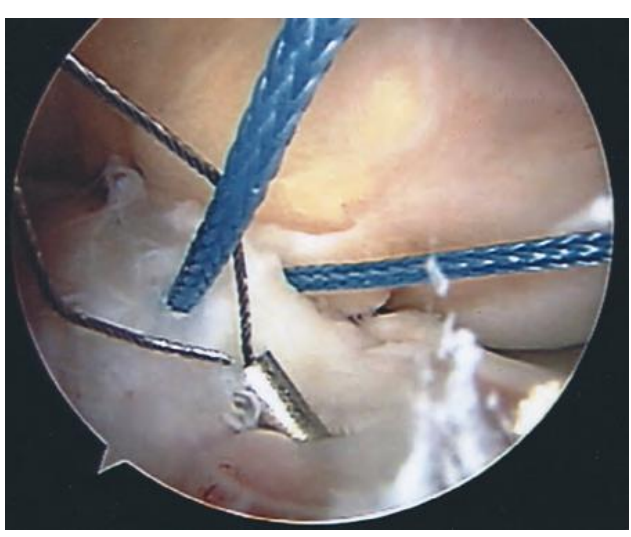

Figura 5: sutura tipo Krakow a nivel distal de LCA, pasando las suturas a través del fragmento y túneles óseos y anudando en cara anteromedial de la metáfisis tibial. 


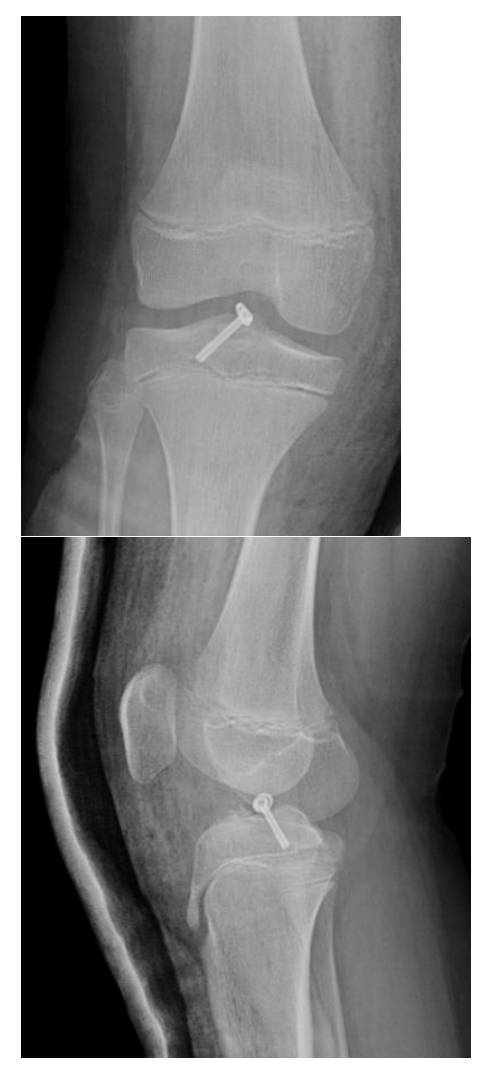

Figura 6: tornillo canuladoosteosintetizando el fragmento osteocondral.

Artroscopia: los portales utilizados habitualmente son el anteromedial y el anterolateral. Una vez visualizada la articulación, es importante localizar el ligamento intermeniscal y el cuerno anterior del menisco medial y desplazarlos para evitar su interposición en el foco de fractura. El uso de una aguja del calibre 18 permite mantener la retracción del ligamento intermeniscal. La reducción de la fractura se realizará mediante la sonda exploradora y el gancho palpador (Figura 7). A continuación, se realizará la osteosíntesis del fragmento (siguiendo los mismos principios de osteosíntesis descritos en la técnica abierta), con las siguientes consideraciones:

Fragmentos osteocondrales mayores de $1 \mathrm{~cm}$ con buen componente óseo: osteosíntesis mediante un tornillo canulado de $20 \mathrm{~mm}$, colocado de tal manera que no afecta la fisis(misma técnica que con la artrotomía). La cabeza del tornillo debe encontrarse en el interior de la sustancia del LCA para evitar pinzamientos en extensión (Figura 8).

Hay que tener en cuenta que la colocación de un tornillo transfisario en pacientes esqueléticamente inmaduros requiere la retirada de material de osteosíntesis en 3 meses para evitar cierre fisario $y$ deformidades en recurvatum.

Fragmentos menores de $1 \mathrm{~cm}$ : el método de elección es la utilización de suturas, siguiendo la técnica descrita en el apartado de "artrotomía" (Figura 5).

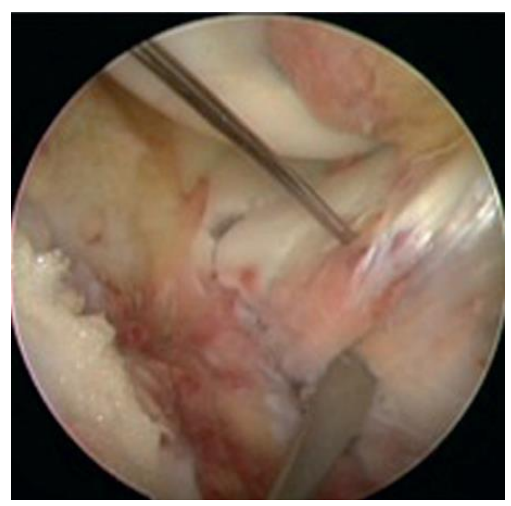

Figura 7: reducción del fragmento por vía artroscópica.

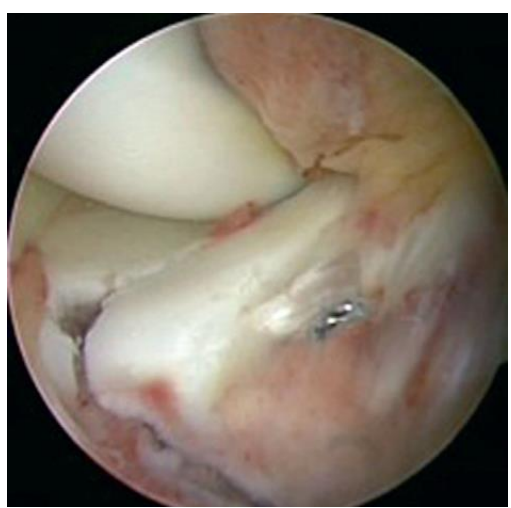


Figura 8: osteosíntesis artroscópica mediante tornillo canulado.

\section{AVULSIONES DEBIDAS A}

\section{ARRANCAMIENTO DE LCP}

Son lesiones poco frecuentes, y el tratamiento depende del grado de desplazamiento ${ }^{(1)}$ :

Lesiones no desplazadas: inmovilización yeso isquiopédico durante 6 semanas.

Lesiones mínimamente desplazadas: existe controversia en cuanto al tratamiento ortopédico mediante inmovilización con yeso o la reducción abierta y fijación interna.

Lesiones desplazadas: reducción abierta y fijación interna. Posteriormente inmovilización con yeso 4 semanas.

En estos casos también se han descrito resultados similares entre la realización de artrotomía (abordaje posterior) o artroscopia. En cuanto a la fijación interna puede realizarse con tornillo canulado si el fragmento es lo suficientemente grande $o$ mediante suturas si el fragmento es pequeño.

\section{PRONÓSTICO Y COMPLICACIONES}

El pronóstico de estas fracturas es bueno en general, sobre todo si se consigue una reducción anatómica de la fractura, la mayoría de los pacientes recuperan un balance articular excelente, y un $85 \%$ de los pacientes son capaces de realizar actividades de alta demanda funcional (1).

Aun así, existen ciertas complicaciones a destacar:

En algunos estudios se ha detectado una falta de extensión completa, preferentemente en fracturas tipo II y III, debido a interposición de fragmento, aunque normalmente se trata de déficits inferiores a $10^{\circ}$. En caso de producir mucha sintomatología, pueden resecarse los fragmentos mal consolidados y reinsertarse

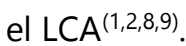

La artrofibrosis es la segunda complicación más frecuentemente observada, debida generalmente a un excesivo tiempo de inmovilización, y existen tasas descritas de hasta un $60 \%$ de paciente con una pérdida mayor o igual a $10^{\circ}$ de rango articular $(2,3,9,12)$.

Otra complicación vista en algunos estudios es la sensación de inestabilidad de rodilla, se ha descrita que hasta el $64 \%$ de pacientes se quejaban de inestabilidad anterior $^{(1,3)}$.

Existen estudios que describen que hasta un $74 \%$ de pacientes ofrecen un pequeño aumento de laxitud en la maniobra de Lachman respecto a la rodilla contralateral. No obstante, muy pocos pacientes referirán sintomatología $a^{(1,2,8,12)}$.

La laxitud puede deberse a una reducción inadecuada del fragmento, que produce una falta de tensión del LCA.

Complicaciones derivadas de roturas no diagnosticadas de LCM o lesiones fisarias concurrentes ${ }^{(1)}$.

\section{BIBLIOGRAFÍA}

Riccio, A.l., Wilson, P.L., Wimberly, R.L. Disorders of the neck. In: J. A. Herring, Tachdjian's Pediatric Orthopaedics. 5th Edition. Texas: Elsevier Saunders; 2014 (pp: 1442-1447).

Adams, A., Talathi, N., Gandhi, J., Patel, N., \&Ganley, T. (2018). Tibial Spine Fractures in Children: Evaluation, Management, and 
Future Directions. The Journal of Knee Surgery, 31(05), 374-381.

Bogunovic, L., Tarabichi, M., Harris, D., \& Wright, R. (2014). Treatment of Tibial Eminence Fractures: A Systematic Review. Journal of Knee Surgery, 28(03), 255-262.

Watts, C.D., Larson, N., Milbrandt, T.A. (2016). Open versus arthroscopic reduction for tibial eminence fracture fixation in children. Journal of Pediatric Orthopaedics, 36:437-439.

Momaya, A. M., Read, C., Steirer, M., \& Estes, R. (2018). Outcomes after arthroscopic fixation of tibial eminence fractures with bioabsorbable nails in skeletally immature patients. Journal of PediatricOrthopaedics B, 27(1), 8-12.

Scrimshire, A. ., Gawad, M., Davies, R., \& George, H. (2018). Management and outcomes of isolated paediatric tibial spine fractures. Injury, 49(2), 437-442.

Osti, L., Buda, M., Soldati, F., Del Buono, A., Osti, R., \&Maffulli, N. (2016). Arthroscopic treatment of tibial eminence fracture: a systematic review of different fixation methods. British Medical Bulletin, 118(1).

Shin, Y.-W., Uppstrom, T. J., Haskel, J. D., \& Green, D. W. (2015). The tibial eminence fracture in skeletally immature patients. Current Opinion in Pediatrics, 27(1), 50-57.

Gans, I., Baldwin, K. D., \& Ganley, T. J. (2013). Treatment and Management Outcomes of Tibial Eminence Fractures in Pediatric Patients. The American Journal of Sports Medicine, 42(7), 1743-1750.

Bomar, J. D., \& Edmonds, E. W. (2016). Surgical Reduction and Fixation of Tibial Spine Fractures in Children. JBJS Essential Surgical Techniques, 6(2), e17.
Ganley, T. J., \&Brusalis, C. M. (2016). Surgical Reduction and Fixation of Tibial Spine Fractures in Children. JBJS Essential Surgical Techniques, 6(2), e18.

Vander Have, K. L., Ganley, T. J., Kocher, M. S., Price, C. T., \& Herrera-Soto, J. A. (2010). Arthrofibrosis after Surgical Fixation of Tibial Eminence Fractures in Children and Adolescents. The American Journal of Sports Medicine, 38(2), 298-301. 